\title{
Study of Mechanics of Physically Transient Electronics: A Step Toward Controlled Transiency
}

\section{Simge Çınar, ${ }^{1}$ Reihaneh Jamshidi, ${ }^{1}$ Yuanfen Chen, ${ }^{1}$ Nastaran Hashemi, ${ }^{1,2,3,4}$ Reza Montazami ${ }^{1,2,3,4}$}

${ }^{1}$ Department of Mechanical Engineering, lowa State University, Ames, lowa, 50011

${ }^{2}$ Ames Laboratory, Department of Energy, Ames, lowa, 50011

${ }^{3}$ Center for Bioplastics and Biocomposites, lowa State University, Ames, lowa, 50011

${ }^{4}$ Center of Advanced Host Defenses Immunobiotics aaand Translational Medicine, lowa State University, Ames, lowa, 50011

Correspondence to: R. Montazami (E-mail:reza@iastate.edu)

Received 18 September 2015; accepted 29 September 2015; published online 00 Month 2015

DOI: 10.1002/polb.23941

ABSTRACT: Transient electronics is a class of electronic devices designed to maintain stable operation for a desired and preset amount of time; and, undergo fast and complete degradation and deconstruction once transiency is triggered. Controlled and programmed transiency in solvent-triggered devices is strongly dependent on chemical and physical interactions between the solvent and the device, as well as those within the device itself, among its constituent components. Mechanics of transiency of prototypical transient circuits demonstrate strong dependence of the transiency characteristics on that of the substrate. In the present study, we demonstrate the control of transiency through the dissolution behavior of a substrate for the devices with electronic parts composed of colloidal units. It is observed that the physical circuit-substrate interactions are the dominating factors in defining the overall transiency behavior of the device. (C) 2015 Wiley Periodicals, Inc. J. Polym. Sci., Part B: Polym. Phys. 2015, 00, 000-000

KEYWORDS: dissolution mechanics; nanoparticles; rheology; swelling; thin films; transient electronics; transiency kinetics; transient polymers
INTRODUCTION Transient electronics is an emerging class of technology representing materials that are able to vanish in a controlled manner when exposed to stimuli. Unlike conventional electronic devices that are designed to operate over the longest possible duration of time, a defining attribute of transient electronics is to operate over a typically short and welldefined duration of time and undergo self-deconstruction and disappear completely when no further needed.

To date, transiency triggered by exposure to light, ${ }^{1}$ heat, ${ }^{2}$ or solvent (often aqueous) ${ }^{3-7}$ is reported. Due to their potential applications in biomedical implants, temporary environmental sensors/monitors, "green" electronics, hardware security, and military applications, aqueous solvent-triggered transient devices are by far the most studied systems. Depending on the application and design of transient electronic devices, transiency can be triggered and stimulated by exposure to water, water-based solvents or bodily fluids (phosphatebuffered saline (PBS), urine, saliva, etc.). Typically, in this class of transient electronic devices water-soluble organic electronic materials ${ }^{6-8}$ are used along with water-soluble metals such as $\mathrm{Mg}$ and $\mathrm{Zn}$ to form electronic components and conductive paths of the circuit; ${ }^{3,9-12}$ less common is use of very small amounts of innately insoluble materials such as silicon which dissolves at a notably slow rate. ${ }^{13,14}$

Polymer-based and natural water-soluble substrates with programmable transiency rate, lasting from minutes to months, are investigated and reported by others and us. In the recent studies, thin films made from silk, ${ }^{3,15}$ poly(L-lactide-co-glycolide) (PLGA), ${ }^{16}$ poly(dimethylsiloxane) (PDMS), ${ }^{17}$ polylactic acid (PLA) ${ }^{18}$ polycaprolactone (PCL), ${ }^{10}$ poly(glycerol-co-sebacate) (PGS), ${ }^{19}$ poly(vinyl alcohol) (PVA), ${ }^{16,20}$ and nanofibrous polymeric membranes ${ }^{21}$ were investigated as substrates of transient electronic devices. In some studies, membranes were investigated separately and in others loaded with electric circuits.

Electronic components are typically fabricated on the substrates by a wide range of fabrication techniques, including physical vapor deposition, electron beam evaporation, photolithography, surface modification, stencil mask printing, and/ or etching of thin films of bulk materials. Transiency

Additional Supporting Information may be found in the online version of this article.

(c) 2015 Wiley Periodicals, Inc. 
mechanisms of these electronic components are investigated both analytically ${ }^{11,14}$ and experimentally. Depending on materials and stimuli, transiency of electronic components occur through corrosion, ${ }^{9}$ dissolution, ${ }^{3,13,14}$ hydrolysis, ${ }^{22}$ or a combination of these processes; ${ }^{11}$ and it is typically quantified by means of mass loss ${ }^{21}$ or change in the thickness ${ }^{13,14}$ of the component. Since these processes all depend on penetration of water or hydroxide ions into the structure through the thickness, the dissolution time and rate are determined by reaction constants, diffusivities of the materials, the thickness of the films, and other parameters of materials and solutions such as materials' morphology, and $\mathrm{pH}$, temperature, and concentration of liquid media. Unlike polymeric substrates that typically have fast transiency rates, transiency of metallic thin films, even for very small thicknesses, when deposited from bulk is very slow and very often a limiting factor to transiency of the system as a whole. If the electronic components, however, could be deconstructed by an external force different from the dissolution, the faster, almost instant, transiency could be achieved; besides, more robust transient electronic systems, where the material choice for electronic components would not be limited to soluble metals, could be possible.

We hypothesized here to use the force generated by swelling of a substrate (swelling force) to enhance physical destruction and transiency of the electronic components; and, as control factor for programming the transiency rate. We report fabrication of conductive path thin films from colloidal metallic particles, rather than bulk materials; which can be easily fabricated on a substrate and redispersed under the effect of swelling force. In such transient electronic devices composed of colloidal particles, transiency can be programmed by means of dissolution/swelling characteristics of substrate and concomitant redispersion of colloidal-based circuit.

In a recent work, ${ }^{23}$ we utilized this technique to fabricate transient soft (flexible and stretchable) bioelectronics; and studied effects of magnitude and frequency of external mechanical force on electrical properties of the strained samples. In the present work, however, we investigate mechanics of transiency and consider correlations between material composition and mechanical force on rate and extent of sample deconstruction. Finding from our recent and current study are complementary and would enable better understanding of soft transient circuits from electrical, chemical, and mechanical point of view.

In this study, PVA is used as a substrate material due to its swelling nature (swelling indices up to $153 \%$ in water, ${ }^{24}$ fast dissolution of its sucrose containing composites, ${ }^{20}$ and versatility in terms of designing its chemical and mechanical properties by felicitous choice of composition. ${ }^{20}$ In situ dissolution/transiency behavior of PVA-based substrates and colloidal silver-based electronic components was investigated through rheological and swelling behaviors, based on physical interactions within/between substrate, electronic components, and the stimuli-water.

\section{RESULTS AND DISCUSSION}

\section{Mechanism of Disintegration}

In order to better predict and control the behavior of transient systems, understanding dissolution behaviors of substrate and disintegration of the electronic components are of significant importance. To this end, the dissolution behaviors of the substrate and device (substrate including the circuit) are scrutinized.

Dissolution behavior of polymers is influenced by the solventmonomer and monomer-monomer interactions. ${ }^{25}$ For transiency to occur, solvent-monomer interactions should dominate over monomer-monomer interactions allowing the solvent to penetrate into the polymer matrix and cause the matrix to swell. For thermoplastics, such as uncrosslinked PVA used in this study, hydrogen bonds are formed between water and hydroxyl groups of PVA, polymer chains disentangle as a result of water uptake, and diffuse out from the matrix. Solution of single polymer chains obtained at the end defines the complete dissolution (Fig. 1, top row). We speculate here that expansion of the membrane applies a mechanical force on the circuit, which results in its physical disintegration. As illustrated in Figure 1 , bottom row, initially (stages 1 and 2), expansion force may not be enough to disintegrate the circuit as integrity of the circuit prevents movement of the polymer chains that are in contact with the circuit, and leads to a resistance to expansion. As the tendency to expansion (swelling force) reaches a critical point, the circuit starts to disintegrate (stage 3). Finally, polymer matrix is completely dissolved; and, due to lack of a mechanical support from the substrate platform, individual colloidal particles forming the circuit are dispersed in the solution (stage 4). The suggested mechanism is tested by means of rheological behavior of dissolution and the swelling behavior of the substrate and the device.

\section{Rheological Behavior of Dissolution}

Rheological properties of polymer solutions are influenced by the polymer structure; thus, viscosity measurements could be utilized to assess the extent of transiency of the polymer membrane. $^{26,27}$ Shear stress $(\tau)$ and viscosity of a polymer solution $(\eta)$ are directly proportional as presented in eq 1 (Newton's law of viscosity).

$$
\tau=\eta \cdot \dot{\gamma}
$$

where $\dot{\gamma}$ is the shear rate applied; thus, viscosity measurements conducted at constant shear rate can be used to interpret the strength of chemical interactions in liquid media.

Figure 2 demonstrates the viscosity measurements taken immediately after exposure to water for substrates and devices (substrate including circuit). As water is penetrating through the polymer matrix, chemical interactions among polymer chains are weaken and swelling occurs, expansion in polymer structure leads to disentanglement of some polymer chains; which, in turn, results in a decrease in the viscosity. As demonstrated in Figure 2(a), for a bare polymer substrate an overall gradual decrease of viscosity was observed upon exposure to the 


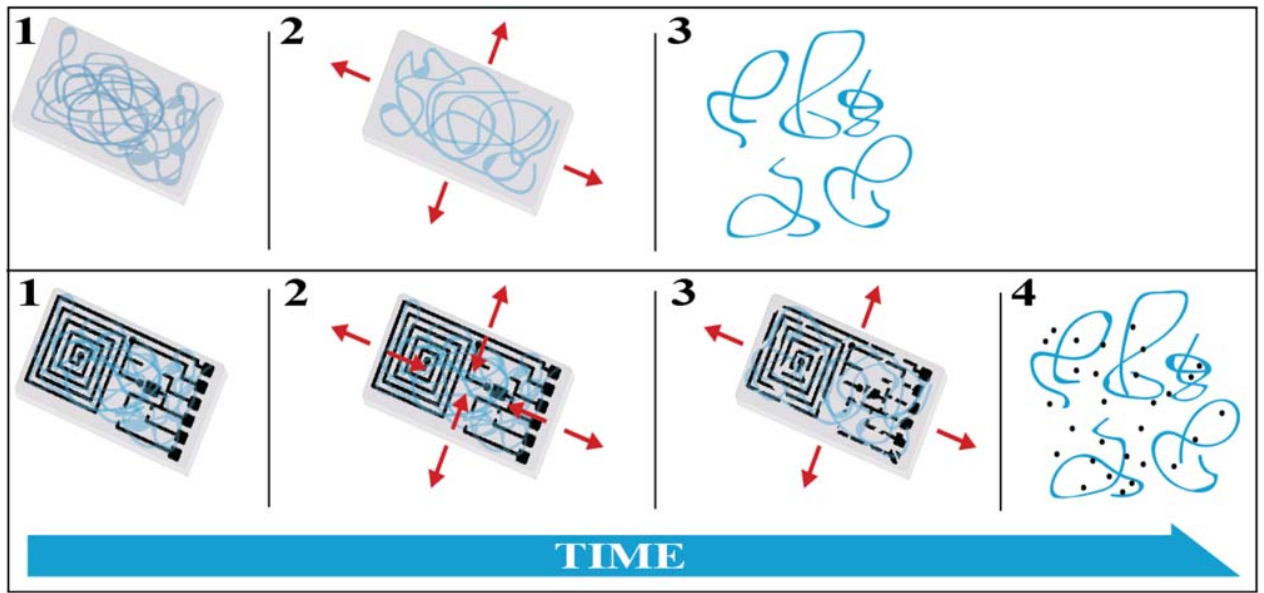

FIGURE 1 Illustration for vanishing of transient electronics based on the dissolution mechanism of polymer membranes. Top row-complete dissolution of polymer matrix: stage 1: polymer film, stage 2,: swelling, stage 3: complete dissolution; bottom row-complete dissolution of transient device: stage 1: device, stage 2: circuit prevents movement of polymer chains connected to the circuit, stage 3: further expansion of substrate and disconnection of circuit, stage 4: complete dissolution of polymer chains and breaking of circuit to individual colloidal particles. [Color figure can be viewed in the online issue, which is available at wileyonlinelibrary.com.]

solvent; and, continued until a stable plateau was reached. Fabrication of an electric circuit on polymer substrate proved to limit mobility of polymer chains against each other when exposed to the solvent; and, delays the overall swelling process. Contrary to bare polymer substrates, those containing a circuit (device) exhibited a two-stage plateau [Fig. 2(b)], the first implying the resistance of the system to swelling at relatively high viscosity followed by a second plateau at approximately the same viscosity of that of a bare substrate indicating redispersion and dissolution of the whole system (circuit and sub-

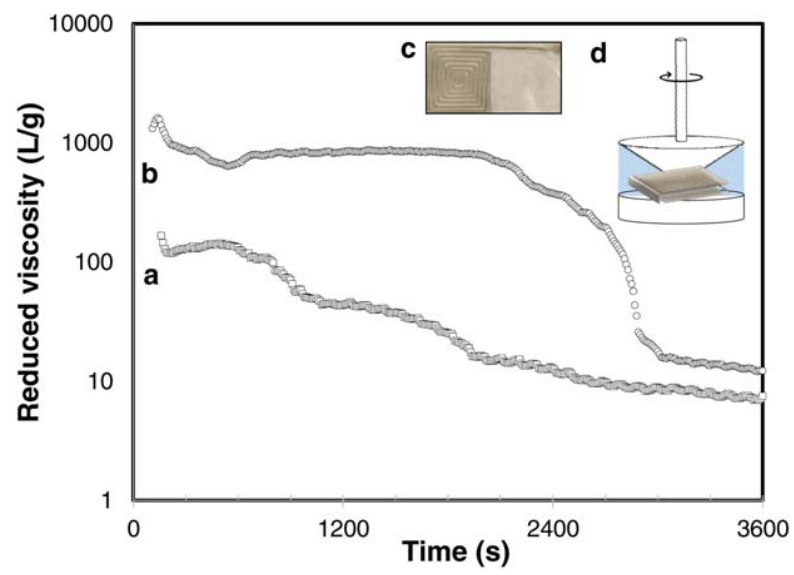

FIGURE 2 Viscosity measurements for PVA polymer matrix substrate (a), and the transient device (b) over the period of time demonstrating their dissolution. Viscosity was given as the reduced viscosity to normalize the measurements with respect to the concentration of a sample. Note that the plot is semilog. PVA/sucrose polymer matrix with the ratio of 1:2 was used with silver-based circuit (c). The typical experimental set-up for viscosity measurement of the device (substrate with circuit on top) is shown (d). [Color figure can be viewed in the online issue, which is available at wileyonlinelibrary.com.] strate). The time separating the two plateaus is the time required for enough amount of solvent to penetrate into the matrix to generate a swelling force large enough to dominated over compression stress due to the presence of circuit; at this point the circuit is disconnected and a steep reduction in viscosity is measured (stage 3 in bottom row of Fig. 1), referred to as break point (bp) for system transiency as the broken circuit expected to lose its function. The second equilibrium state is an intermediate step where the shape of the circuit is partially preserved although the colloidal particles are disconnected. In the third and last equilibrium state, viscosity approached to the equilibrium indicating a stable partially or fully dissolved state (stage 4 in bottom row of Fig. 1). Absence of a platform to hold individual colloidal particles leads to complete disintegration of the circuit and redispersion of constituent colloidal particles. Supporting Information Figure S1 demonstrates the viscosity measurements for varying dissolution rates and provides a set of images collected after the measurements justifying use of viscosity measurements in investigation of the dissolution behavior of polymer substrates and devices.

The break-point time $\left(t_{\mathrm{bp}}\right)$, the initial reduced viscosity $\left(\eta_{0}\right)$, and the reduced break-point viscosity $\left(\eta_{\mathrm{bp}}\right)$, were determined as the critical parameters of dissolution obtained from viscosity measurements to investigate the effect of dissolution behavior of substrates and transient devices (Supporting Information Fig. S2).

\section{Vanishing Behavior of Transient Substrates and Electronics Rheological Behavior}

The critical parameters of transient behavior are compared for varying concentration of the PVA/sucrose substrates in the absence (substrate) and the presence of circuit (device) and reported in Figure 3 and Supporting Information Table S1. Figure 3(a) shows that sucrose dominating substrates, 

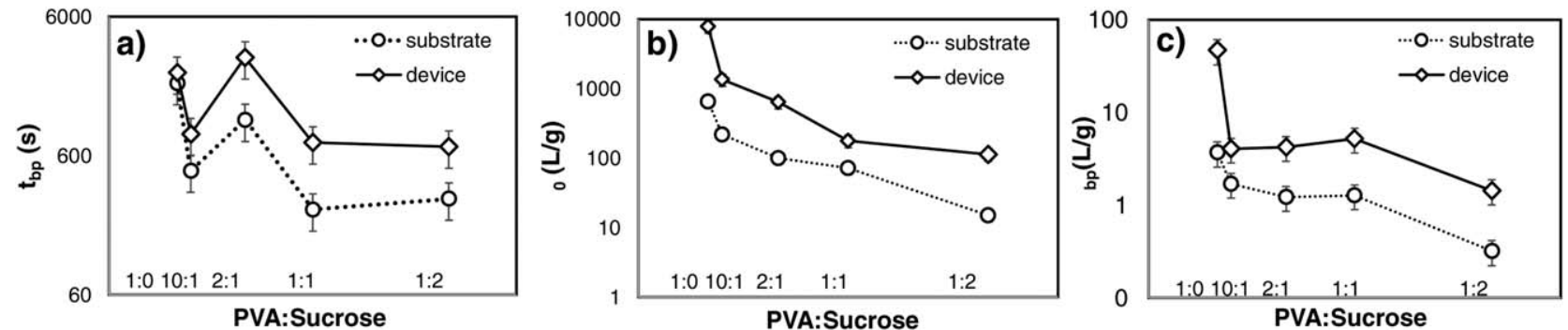

FIGURE 3 The effect of sucrose content of polymer matrix substrate on the critical parameters of dissolution obtained from viscosity measurements for substrates (dotted lines) and devices which include circuit on substrate (solid lines). $t_{\mathrm{bp}}$ is the break-point time for system transiency (a), $\eta_{0}$ is the initial reduced viscosity (b), and $\eta_{\mathrm{bp}}$ is the reduced break-point viscosity (c).

the substrates with sucrose concentration of equal to or higher than that of PVA, manifest faster dissolution than others. Similar behavior was observed in our previously reported study ${ }^{20}$ by measuring the weight loss of substrates as a function of time. Comparing to weight loss measurements, viscosity measurements are expected to result in relatively longer dissolution times since the available water content for dissolution is limited (the volume of cone and plate geometry is about $1.2 \mathrm{~mL}$, regarding concentration of the samples are around 13-17 g/L). In the case of pure PVA, for instance, PVA could not fully dissolved due to the lack of enough solvent (Supporting Information Fig. S1a), thus exhibited much higher viscosity value at plateau region with the lack of sharp decrease in viscosity.

Faster dissolution and lower viscosities of sucrose containing PVA substrates, the ones containing sucrose content equal to or higher than that of PVA, could be explained by chemical interactions within substrate and between substrate and the solvent. The full FTIR spectra and the detailed chemical assignments of the bands are presented in Supporting Information (Fig. S3 and Table S2). The FTIR spectra in Figure 4 focuses on the band at $1141 \mathrm{~cm}^{-1}$ corresponding to the symmetric $\mathrm{C}-\mathrm{C}$ stretching mode and shows pronounced absorption with increased sucrose concentration, particularly as the content of the sucrose equal to or higher than that of PVA, indicating the more crystalline polymer substrates. Mallapragada and Peppas ${ }^{28}$ reported that dissolution rate of the PVA polymer films are proportional to the degree of crystallinity; thus, fast dissolution of sucrose dominating PVA substrates is attributed to their increasing crystallinity. With increasing sucrose concentration, more sucrose interacts with higher portion of PVA chains create more volume of crystalline domains while intuitively disentangling the polymer chains. Combined with very fast dissolution of sucrose domains in water, less entangled matrix leads to faster dissolution and lower viscosities as observed in Figure 3(a-c).

As the polymer matrix is first exposed to water, the measured viscosity can be considered as an analogous to the Young's modulus, as the measured value is the shear stress in the case of viscosity measurements rather than the tensile stress as in tensile measurements. In order to calculate the reduced viscosity, the increment in viscosity with respect to pure water solvent is calculated and normalized to the sub- strate concentration in water. The Young's modulus values, which have been recently reported for the PVA/sucrose composites, ${ }^{20}$ trends in the same way with the initial reduced viscosity reported in Figure 3(b), which partly confirms the validity of using viscosity measurements to investigate the dissolution behavior. Having circuit on top of the substrate resulted in higher viscosity, that is, exhibits higher resistance to flow, comparing to the measurements for substrates with the same composition.

Sucrose acted as a plasticizer for PVA and led to decrease in the break-point viscosity of substrate solutions from 3.7 to $0.3 \mathrm{~L} \mathrm{~g}^{-1}$ [Fig. 3(c) and Supporting Information Table S1]. Particles or broken pieces of circuits in solution act as obstacles to flow of solution and lead to higher reduced

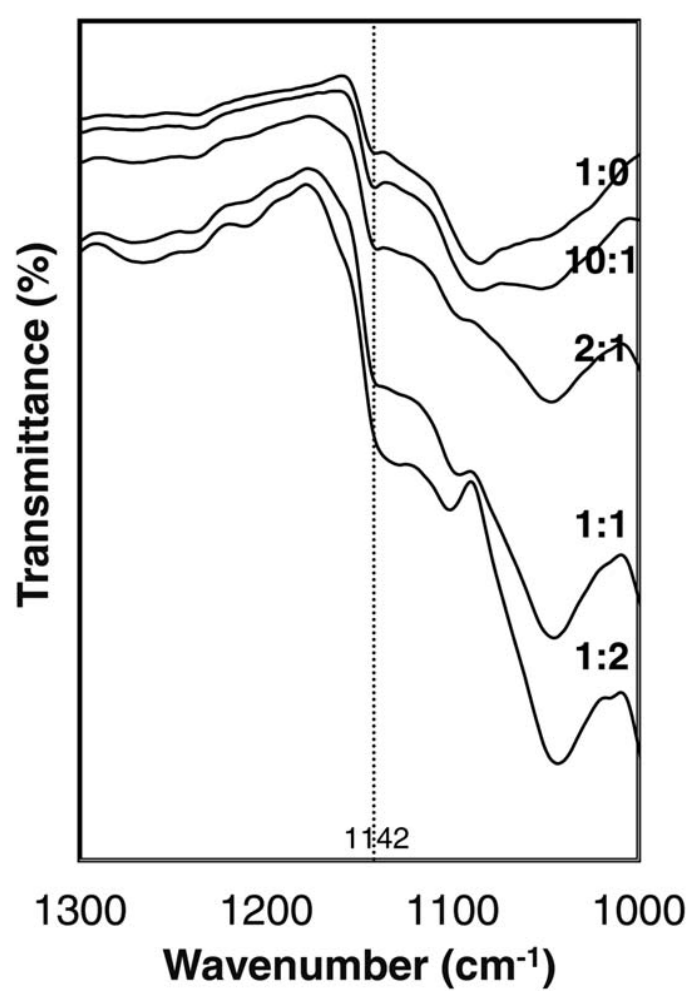

FIGURE 4 ATR-FTIR spectra of PVA-sucrose substrates with varying composition. The bands at $1142 \mathrm{~cm}^{-1}$ are the indication of the semicrystalline PVA. 
break-point viscosity values than the viscosities of regarding substrates with the same concentration but without circuit [Fig. 3(c) and Supporting Information Table S1]. In the presence of circuit (device), the break-point viscosity of the entire solution decreased from 47.0 to $1.4 \mathrm{~L} \mathrm{~g}^{-1}$ [Fig. 3(c) and Supporting Information Table S1].

Reported results support that rheological measurements can be used as a tool for investigation the dissolution behavior of transient devices since it explains the full in situ dissolution behavior of not only substrates, but also the devices (substrates with circuit). Examples of its further use include mimicking the real application conditions of transiency in a sample such as in solvents with varying $\mathrm{pH}$, temperature, and chemistry. The critical parameters of application in interest such as the maximum shear stress, dissolution behavior under shear, etc., could also be investigated by means of the rheological behavior, which is challenging via the weight loss measurements. Viscosity measurements at constant shear rate offer comprehensive information about not only in situ behavior of substrates, but also that of devices as a whole, such as dissolution, degradation and/or transiency behavior, viscoelastic behavior, and programmability.

\section{Swelling Behavior}

Function of a transient device is directly related with integrity of a circuit. Since disconnection and/or breaking of the circuit was expected to be controlled by swelling a polymer composite film, expansion of the substrates and devices was investigated. Figure 5 demonstrates the three distinct behaviors of devices triggered by the swelling force applied in a certain time span, which could be controlled only by changing the composition of the substrate. Among all, the device with pure PVA substrate showed the slowest expansion while the one with 2:1 PVA:sucrose ratio [Fig. 5(b)] exhibited the largest expansion. Independent from the duration of expansion, in both cases, expansion forces lead to breaking of the circuit into almost individual particles. As the concentration increased further [to 1:1 PVA:sucrose ratio, Fig. 5(c)], however, the transiency of substrate was too fast, thus the substrate dissolved even before breaking the circuit, left it almost complete.

Figure 5(d) compares the pace of expansion for the blank and the circuit parts of devices in the first minute of an experiment. It is known from viscosity measurements reported above that interactions of PVA polymer chains in pure PVA substrate are relatively much stronger (deduced by the higher viscosity values) while its dissolution is dependent on the sucrose concentration: the dissolution is much slower for substrates with sucrose concentration equal to or higher than that of PVA (deduced by the higher dissolution times). During swelling experiments, expansion of pure PVA substrates reached to the values as high as $60 \%$ in $60 \mathrm{~min}$ without a significant difference between the substrate and the circuit containing parts of the same device (the difference is about $10 \%$ at its maximum) indicating that the interactions between polymer chains is strong enough to disconnect the circuits as the film expands. Since the polymer film dissolved slowly, the shape of the circuit could be preserved even after an hour of exposure to stimuli, but in the form of almost individual particles.

With a small addition of sucrose to the PVA (10:1), the largest expansion for the circuit containing part of the device, around $85 \%$ for the blank and $80 \%$, could be obtained in 10 min of exposure [Fig. 5(a)]. Strong chemical interactions (hydrogen bonding capability) of sucrose with both PVA and water might attract the water molecules towards into the polymer matrix and results in larger expansion as compared to that of pure PVA sample. As observed in Figure 5(b), the fast expansion resulted in disconnection of the circuit very fast.

The samples of substrates with the composition of 2:1 exhibits the fastest expansion among other samples [Fig. 5(d)]. The substrate and the circuit containing device parts for this composition expands about 75 and $60 \%$, respectively only in 5 min exposure to stimuli [Fig. 5(b)]. The expansion difference between substrate and the circuit containing parts was decreased from 40 to $20 \%$ in the first minute of exposure indicating that the circuit could not resist to this significant expansion and disconnected in only few minutes after being exposed to the stimuli, thus is expected to exemplify instant transiency.

Increasing the concentration of sucrose to equal or higher concentrations than that of PVA (1:1 and 1:2), resulted in very fast dissolution of substrates. As discussed above, the relatively less entanglements in sucrose dominating substrates due to their higher crystallinity limit the expansion of the composite matrix. Substrates with high sucrose concentration $(1: 1,1: 2)$ could expand to a limited level of $45 \%$ while the maximum expansion of circuit parts was about $20 \%$ [Fig. 5(c)]. Because of the absence of strong expansion forces, the interactions which hold silver particles together dominates, and the circuit could preserve its integrity even though the substrates dissolved away. Such a device could be used where the substrate is critical only to support the device mechanically and holding the components together during its transfer, but required to be discarded after the transfer to enhance the contact between the electronic components and the surface of interest, but does not necessarily requires the transiency.

The mechanical fracture observed in Figure 5(c) could be prevented by adjusting the thickness of the substrate or the active components. Fracture of a circuit due to the bending of a flexible substrate could be prevented by using thinner substrates or thicker circuits as suggested by Hwang et al. ${ }^{10}$ or by optimizing the mechanical, chemical, and transiency properties of a substrate as suggested by Acar et al. ${ }^{20}$ or in the presence of external support such as tissue or skin.

The fact that the integrity of electronic components, thus transiency behavior of the device, could be controlled by dissolution behavior of the substrate as discussed in Figure 5, 


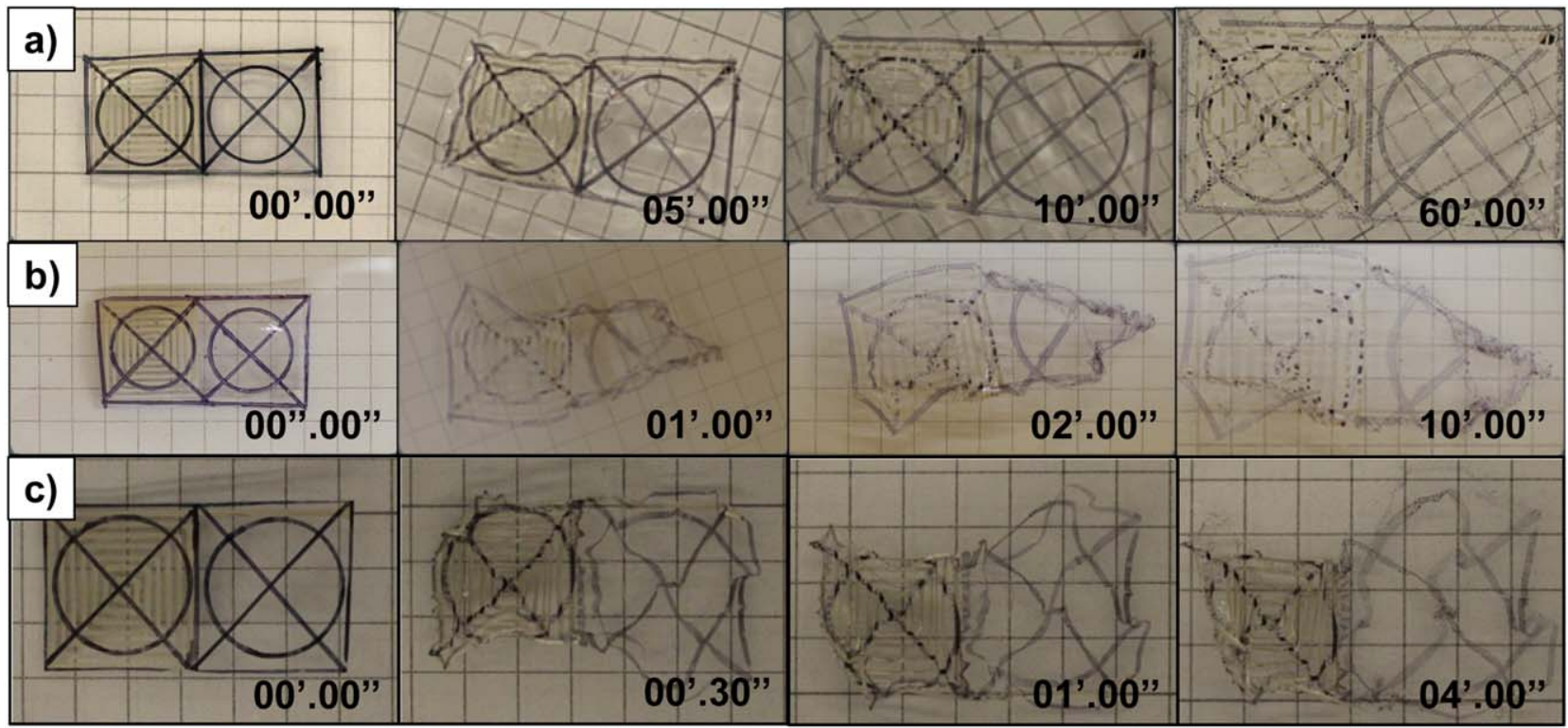

d)

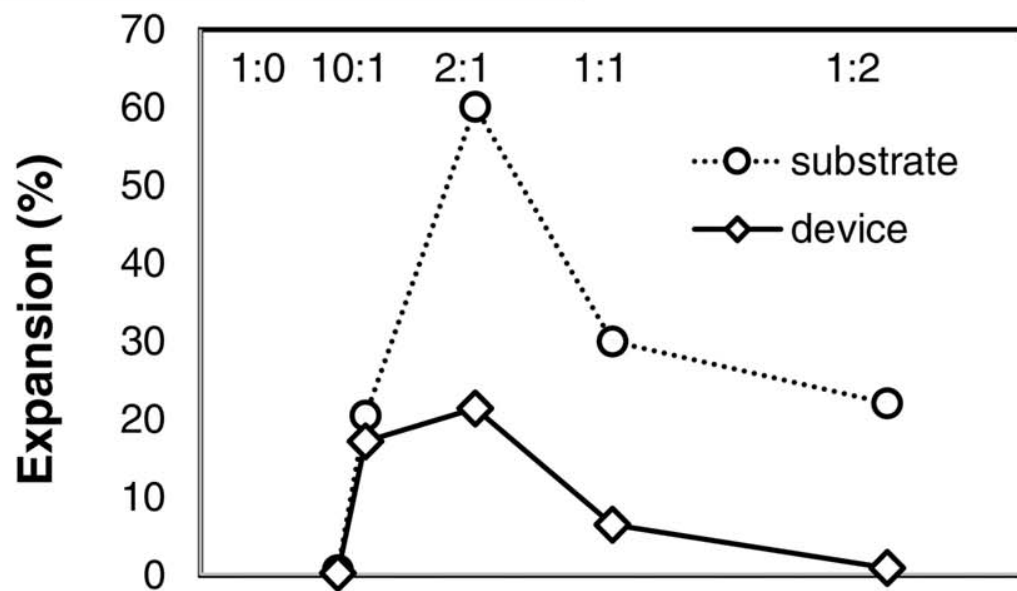

\section{PVA:Sucrose}

FIGURE 5 Swelling of transient devices with PVA/sucrose matrix substrates. PVA:sucrose ratio of 1:0 (a), 2:1 (b), 1:1 (c). Background is a $5 \mathrm{~mm}$ grid. Time passed after immersion of transient devices in water is given at the right bottom corner of images. For all cases $(a-c)$, the images from left to right represents the initial condition, the resistance to swelling in the presence of circuit (please compare the sides with circuit and the one of substrate), the circuit after the break point, and the final appearance of the device (after this point, the data was kept recorded, but the change observed was not significant.), respectively. Note that the expansion is not same for each case. (d) Comparison of expansion for the substrate and the circuit containing (device) parts after a minute of exposure to water. Here, the error in expansion stemmed from the physical contact of the substrate and the circuit containing part is assumed negligible; thus, the values should be considered relatively. [Color figure can be viewed in the online issue, which is available at wileyonlinelibrary.com.]

the same simple system can be used for a range of applications by felicitous choice of substrate chemistry that tunes the chemistry and physical properties of the devices. Slowly degrading substrates (1:0 and 10:1) can be used for biological application which requires programmable degradation, while instant degradation as in the sample of 2:1 can be used for military applications. The samples which the circuit could preserve its integrity despite of the degradation of substrate $(1: 1,1: 2)$ can be used for making sensors applied on surfaces of human skin, plants, etc., for more accurate and precise measurements. After placing the sample on the surface, substrate could be washed off. The remaining functional parts of the device would directly interact with the surface of a target and will potentially enhance quality of a contact. $^{4}$

\section{CONCLUSIONS}

The results presented here provide a scientific foundation for a new approach toward fabrication of conductive components of transient electronics, utilizing colloidal metal particles to achieve fast, and coordinated deconstruction and 
transiency of polymeric substrate and electronic components in transient electronic devices. A transformative and appealing aspect of this approach is that it allows realization of controlled transiency by eliminating limiting factors posed by dissolution rate of metals. It was demonstrated that if colloidal metal particles are used for conductive paths, the transiency of polymer-based substrates, which are easier to control, can be used to program transiency of the whole system; and, that unlike conventional transient electronics, transiency rate of substrate can be a controlling and dominating factor.

\section{EXPERIMENTAL}

\section{Preparation of Substrates}

Poly (vinyl alcohol) (PVA) (Mowiol 10-98, MW: 61,000 g $\mathrm{mol}^{-1}$, 98.0-98.8 mol \% hydrolysis) and sucrose were purchased from the Sigma-Aldrich and used as received. To prepare polymer membranes with different PVA:sucrose ratio, desired amount of sucrose was dissolved in $20 \mathrm{~mL}$ deionized water (resistivity $\geq 18.0 \mathrm{M} \Omega \mathrm{cm}$ ) at room temperature. PVA and $50 \mu \mathrm{L}$ of $1 \mathrm{M}$ aqueous $\mathrm{HCl}$ solution was added to the solution. The solution was magnetically stirred at $70{ }^{\circ} \mathrm{C}$ for $4 \mathrm{~h}$ to allow complete dissolution of PVA, then cooled to room temperature and casted on $86 \mathrm{~mm} \times 128 \mathrm{~mm}$ flat polymer molds. Polymer films were carefully peeled off when dried; drying time varied from 24 to $48 \mathrm{~h}$ depending on the sucrose concentration and film thickness. For $1 \mathrm{~g}$ total substrate, the films thickness was $70 \pm 10 \mu \mathrm{m}$. Films with PVA:sucrose ratios of 1:0, 10:1, 2:1, 1;1, 1:2) were fabricated.

\section{Fabrication of Circuits}

Conductive silver paste (Pelco, 187 Series) was purchased from Ted Pella and diluted with acetone at 1:1 ratio. Vinyl masks with desired circuit design were fabricated using a vinyl cutter (US-Cutter. SC series) with $25 \mu \mathrm{m}$ resolution and $125 \mu \mathrm{m}$ repetition accuracy. Conductive silver paths were produced by spray coating of diluted silver over the vinyl mask. An average circuit consisted of $20 \mathrm{~mm}$ wide conductive paths and covered an area of $1.25 \mathrm{~cm}^{2}$ on the substrate. The net amount of silver (dried) used to cover this area was $2.8 \pm 0.2 \mathrm{mg}$ (corresponding thickness is about $13 \mu \mathrm{m}$ ).

\section{Rheology Measurements}

Viscosity of the substrates and devices were measured by a rheometer (AR2000ex, TA Instruments) with a cone and plate geometry (steel $4^{\circ}$ cone angle and $40 \mathrm{~mm}$ diameter). Specimen was placed in $1.17 \mathrm{~mL}$ of DI water at center of the plate. A solvent trap was used to prevent water evaporation during measurements. Viscosity of the sample was measured and recorded every $10 \mathrm{~s}$ at the constant shear rate of $1 \mathrm{~s}^{-1}$ at $25.0 \pm 0.1{ }^{\circ} \mathrm{C}$.

The recorded viscosity values were normalized with respect to the solvent viscosity (pure water); the concentration of the solution versus the reduced viscosity, $\eta_{\text {red, }}$ were then plotted. Reduced viscosity was deduced from the viscosity of solution $(\eta)$, solvent $\left(\eta_{0}\right)$, and concentration $(c)$, using eq 2 .

$$
\eta_{\text {red }}=\frac{\eta / \eta_{0}-1}{c}
$$

Data were shifted on the horizontal axis to correct for the few second time lag between immersing the sample in the solvent and starting the analysis.

At the applied shear rate of $1 s^{-1}$, the data were fluctuating at every $90 \mathrm{~s}$ indicating the inhomogeneity of the solution during dissolution event, then moving average of 9 was employed to smoothen the data.

\section{Swelling Measurements}

Swelling of polymeric substrates in solvent was quantified by measuring the changes in planar dimensions of the specimens (blank of circuit parts of the device) as a function of time. Specimens were labeled with marks of known geometry and dimensions [Fig. 4(a-c) as an example]. A charged couple device (Canon EOS Rebel SL1 100D) was used to obtain sequential images of specimens against a gridded background at desired time intervals to monitor and record position of the labels and determine swelling rate and behavior. Image J software was used for image analysis. At least three readings were taken for each measurement and averages were reported.

\section{Infrared Spectroscopy}

Attenuated total reflectance-Fourier transform infrared (ATRFTIR) spectroscopy (Frontier Perkin Elmer) equipped with single reflection ATR attachment with diamond crystal was used for this study. Samples were placed directly on the ATR crystal. Four scans with a spectral resolution of $4 \mathrm{~cm}^{-1}$ were taken at room temperature for each sample. Data were processed by Spectrum 10 software. For each spectrum, an interactive baseline correction with respect to the position of $4000 \mathrm{~cm}^{-1}$ was employed.

\section{ACKNOWLEDGMENTS}

This work was supported in part by Iowa State University PIIR (Presidential Initiative for Interdisciplinary Research) grant. The authors would like to thank Professor Mufit Akinc for his support of this work.

\section{REFERENCES AND NOTES}

1 H. L. Hernandez, S. K. Kang, O. P. Lee, S. W. Hwang, J. A. Kaitz, B. Inci, C. W. Park, S. Chung, N. R. Sottos, J. S. Moore, J. A. Rogers, S. R. White, Adv. Mater. 2014, 26, 7637-7642.

2 C. W. Park, S. K. Kang, H. L. Hernandez, J. A. Kaitz, D. S. Wie, J. Shin, O. P. Lee, N. R. Sottos, J. S. Moore, J. A. Rogers, S. R. White, Adv. Mater. 2015, 27, 3783-3788.

3 S. W. Hwang, H. Tao, D. H. Kim, H. Cheng, J. K. Song, E. Rill, M. A. Brenckle, B. Panilaitis, S. M. Won, Y. S. Kim, Y. M. Song, K. J. Yu, A. Ameen, R. Li, Y. Su, M. Yang, D. L. Kaplan, M. R. Zakin, M. J. Slepian, Y. Huang, F. G. Omenetto, J. A. Rogers, Science 2012, 337, 1640-1644.

4 S. W. Hwang, D. H. Kim, H. Tao, T. Kim, S. Kim, K. J. Yu, B. Panilaitis, J. W. Jeong, J. K. Song, F. G. Omenetto, Adv. Funct. Mater. 2013, 23, 4087-4093. 
5 S. W. Hwang, G. Park, H. Cheng, J. K. Song, S. K. Kang, L. Yin, J. H. Kim, F. G. Omenetto, Y. Huang, K. M. Lee, J. A. Rogers, Adv. Mater. 2014, 26, 1992-2000.

6 M. Irimia-Vladu, Chem. Soc. Rev. 2014, 43, 588-610.

7 M. Irimia-Vladu, E. D. Głowacki, G. Voss, S. Bauer, N. S. Sariciftci, Mater. Today, 2012, 15, 340-346.

8 M. Irimia-Vladu, N. S. Sariciftci, S. Bauer, J. Mater. Chem. 2011, 21, 1350-1361.

9 L. Yin, H. Cheng, S. Mao, R. Haasch, Y. Liu, X. Xie, S. W. Hwang, H. Jain, S. K. Kang, Y. Su, Adv. Funct. Mater. 2014, 24, 645-658.

10 S. W. Hwang, J. K. Song, X. Huang, H. Cheng, S. K. Kang, B. H. Kim, J. H. Kim, S. Yu, Y. Huang, J. A. Rogers, Adv. Mater. 2014, 26, 3905-3911.

11 R. Li, H. Cheng, Y. Su, S. W. Hwang, L. Yin, H. Tao, M. A. Brenckle, D. H. Kim, F. G. Omenetto, J. A. Rogers, Y. Huang, Adv. Funct. Mater. 2013, 23, 3106-3114.

12 M. Luo, A. W. Martinez, C. Song, F. Herrault, M. G. Allen, Microelectromech. Syst. J. 2014, 23, 4-13.

13 S. W. Hwang, G. Park, C. Edwards, E. A. Corbin, S. K. Kang H. Cheng, J. K. Song, J. H. Kim, S. Yu, J. Ng, J. E. Lee, J. Kim, C. Yee, B. Bhaduri, Y. Su, F. G. Omennetto, Y. Huang, R. Bashir, L. Goddard, G. Popescu, K. M. Lee, J. A. Rogers, ACS Nano 2014, 8, 5843-5851.

14 S. K. Kang, S. W. Hwang, H. Cheng, S. Yu, B. H. Kim, J. H. Kim Y. Huang, J. A. Rogers, Adv. Funct. Mater. 2014, 24, 4427-4434.

15 H. Tao, M. A. Brenckle, M. Yang, J. Zhang, M. Liu, S. M. Siebert, R. D. Averitt, M. S. Mannoor, M. C. McAlpine, J. A. Rogers, D. L. Kaplan, F. G. Omenetto, Adv. Mater. 2012, 24, 1067-1072.
16 C. J. Bettinger, Z. Bao, Adv. Mater. 2010, 22, 651-655.

17 X. Huang, Y. Liu, H. Cheng, W. J. Shin, J. A. Fan, Z. Liu, C. J. Lu, G. W. Kong, K. Chen, D. Patnaik, S. H. Lee, S. Hage-Ali, Y. Huang, J. A. Rogers, Adv. Funct. Mater. 2014, 24, 3846-3854.

18 G. Mattana, D. Briand, A. Marette, A. V. Quintero, N. F. de Rooij, Org. Electr. 2015, 17, 77-86.

19 Y. J. Kim, S. E. Chun, J. Whitacre, C. Bettinger, J. Mater. Chem. B 2013, 1, 3781-3788.

$20 \mathrm{H}$. Acar, S. Çınar, M. Thunga, M. R. Kessler, N. Hashemi, R. Montazami, Adv. Funct. Mater. 2014, 24, 4135-4143.

21 A. H. Najafabadi, A. Tamayol, N. Annabi, M. Ochoa, P. Mostafalu, M. Akbari, M. Nikkhah, R. Rahimi, M. R. Dokmeci, S. Sonkusale, B. Ziaie, A. Khademhosseini, Adv. Mater. 2014, 26, 5823-5830.

22 L. Yin, A. B. Farimani, K. Min, N. Vishal, J. Lam, Y. K. Lee, N. R. Aluru, J. A. Rogers, Adv. Mater. 2015, 27, 1857-1864.

23 R. Jamshidi, S. Çinar, Y. Chen, N. Hashemi, R. Montazami, J. Polym. Sci. Part B: Polym. Phys. 2015, 53, 1603-1610.

24 J. Krzeminski, H. Molisak-tolwinska, J. Macromol. Sci. A: Chem. 1991, 28, 413-429.

25 B. A. Miller-Chou, J. L. Koenig, Prog. Polym. Sci. 2003, 28, 1223-1270.

26 M. Bercea, S. Morariu, D. Rusu, Soft Matter. 2013, 9, 1244-1253.

27 B. Briscoe, P. Luckham, S. Zhu, Polymer 2000, 41, 3851-3860.

28 S. K. Mallapragada, N. A. Peppas, J. Polym. Sci. Part B: Polym. Phys. 1996, 34, 1339-1346. 